\title{
Editorial
}

\section{Liver Biopsy at the Time of Bariatric Surgery: A Benefit for Patients and the Medical Community}

\author{
Elizabeth $C$. Verna, MD, MS ${ }^{1}$ \\ ${ }^{1}$ Division of Digestive and Liver Diseases, Center for Liver Disease and \\ Transplantation, Columbia University College of Physicians and \\ Surgeons, New York, New York
}

Semin Liver Dis 2014;34:1-6.

The growth of the field of hepatology over the past 30 years has largely been driven by the burgeoning incidences and prevalences of two diseases: hepatitis $C$ and nonalcoholic fatty liver disease (NAFLD). This issue of the Seminars in Liver Disease, guest-edited by Professor Jean-Michel Pawlotsky, is devoted principally to the recent explosion of knowledge about the biology of the hepatitis $\mathrm{C}$ virus (HCV) and the resulting appearance of multiple new drugs that dramatically increase the success of HCV treatment. In addition, an editor's choice article from the Longitudinal Assessment of Bariatric Surgery (LABS) Consortium raises important questions about NAFLD.

NAFLD is a common and potentially life-threatening obesity-related comorbidity. Nonalcoholic steatohepatitis (NASH), one component of the widely accepted NAFLD spectrum, may be the most common form of chronic hepatitis in the United States, and has been projected to replace hepatitis C-related liver disease as the leading indication for liver transplant., ${ }^{1,2}$ Bariatric surgery (BS) patients are among the highest risk population for NAFLD, which affects up to $98 \%$ of patients in some series, and the possible use of BS as a therapeutic approach to treat NAFLD is gaining attention. ${ }^{3}$ However, the natural history of NAFLD in BS patients and the impact of BS on NAFLD disease progression remain incompletely described. The depth of our uncertainty about key aspects of fatty liver disease is reflected in recent publications challenging the basic concepts that simple hepatic steatosis is part of the broader spectrum that includes NASH, NASH with fibrosis, and cirrhosis, ${ }^{4}$ or that the proportion of total liver transplants going to patients with fatty liver disease is significantly increasing. ${ }^{5}$

In this issue of the Seminars in Liver Disease, Kleiner and colleagues ${ }^{6}$ report histologic findings from intraoperative liver biopsies in a subset of patients in the LABS study cohort. While biopsies were not performed universally or in an unbiased manner, the burden of liver disease in this population was predictably high, with borderline or definitive NASH found in over a third of biopsied patients and bridging fibrosis or cirrhosis in $4.2 \%$. Perhaps most striking was that among patients with alanine aminotransferase (ALT) $<35 \mathrm{IU} / \mathrm{L}$, a widely accepted upper limit of normal, $24 \%$ had borderline or definitive steatohepatitis, $6.4 \%$ had a NAFLD activity score $(\mathrm{NAS}) \geq 5$, and $2 \%$ had bridging fibrosis or cirrhosis. When even more stringent ALT criteria were used (normal: < 30 IU/ L for men and $<19 \mathrm{IU} / \mathrm{L}$ for women), ${ }^{7} 19 \%$ had borderline or definitive steatohepatitis, $3.7 \%$ had NAS $\geq 5$, and $1.6 \%$ had bridging fibrosis or cirrhosis, rendering even these cutoffs for ALT inadequate to exclude clinically important liver disease in these very high-risk patients. The authors then used logistic regression modeling as a crude indicator of how much liver disease may have gone undiagnosed in the patients who were not biopsied; the models suggested that almost $6 \%$ of unbiopsied patients have bridging fibrosis or cirrhosis and almost half of the patients have borderline or definitive steatohepatitis. As visual inspection of the liver by the surgeon in the operating room may be insufficiently sensitive to detect significant hepatic pathology, much of the important liver disease in the cohort remains undiagnosed.

This report reminds us of just how little we know about the burden of liver disease in the BS population, and perhaps supports the growing sentiment that liver biopsy at the time of BS should be universally considered. Several lines of evidence support the fact that a clearer understanding of the liver histology in BS patients will benefit both individual patients and the medical community.

\section{NALFD Diagnosis and Staging Is an Important Goal}

The diagnosis of steatohepatitis and/or advanced fibrosis in the setting of fatty liver infiltration has important prognostic and therapeutic implications for patients. It is clear that advanced
Address for correspondence Elizabeth C. Verna, MD, MS, Division of Digestive and Liver Diseases, Center for Liver Disease and Transplantation, Columbia University College of Physicians and Surgeons, 622 West 168th Street, New York, NY 10032 (e-mail: ev77@columbia.edu).
Issue Theme New Hepatitis C Therapies; Guest Editor, JeanMichel Pawlotsky, MD, PhD
Copyright @ 2014 by Thieme Medical Publishers, Inc., 333 Seventh Avenue, New York, NY 10001, USA. Tel: +1(212) 584-4662.
DOI http://dx.doi.org/ 10.1055/s-0034-1371549. ISSN 0272-8087. 
fibrosis is the histologic finding most predictive of overall and liver-related mortality among patients with NAFLD, ${ }^{8-10}$ and liver enzymes are not sensitive or specific indicators of advanced fibrosis (as detailed later). The identification of patients with advanced fibrosis is key to the prevention of life-threatening complications including hepatocellular carcinoma (HCC) and/or decompensated end-stage liver disease. It is also clear that patients with NASH have a worse prognosis than those with simple steatosis. Patients with obesity-associated NASH are at risk for fibrosis progression with the development of cirrhosis in $20 \%$ to $40 \%$ of cases, and thus a subsequent risk of hepatic decompensation and HCC. Patients with NASH also have increased liver- and cardiovascular-related mortality compared with matched controls without NAFLD or NAFLD without associated steatohepatitis. ${ }^{11-13}$

In addition to informing prognosis and the need for screening, the identification of NAFLD and/or NASH may provide an opportunity to alter the natural history of disease. While currently recommended therapies are limited to life style modifications, control of metabolic syndrome manifestations, and limited pharmacologic interventions such as vitamin $\mathrm{E}$ and pioglitazone in subsets of patients with biopsy-proven disease, treatment approaches for this increasingly prevalent disease are rapidly evolving. ${ }^{3,14}$ Without knowledge of their chronic liver disease, BS patients with NAFLD and their care providers will increasingly miss important opportunities to halt or slow disease progression. There is an important analogy to hepatitis $\mathrm{C}$, which will remain a major clinical problem for decades despite the new availability of curative treatments because the vast majority of infected individuals worldwide are unaware that they are infected and will not present for treatment until their disease is advanced and symptomatic.

\section{Liver Disease Negatively Impacts Bariatric Surgery Postoperative Outcomes}

While the rates of hepatic decompensation after BS are low overall, it is clear that cirrhosis negatively impacts multiple important patient outcomes including mortality, and that postoperative liver-related deaths do occur. ${ }^{15,16}$ In a recent evaluation of the Nationwide Inpatient Sample dataset between 1998 and 2007, ICD-9 codes were used to compare BS outcomes in patients with (1) no evidence of liver disease, (2) compensated liver disease, and (3) decompensated liver disease (with ascites and/or variceal bleeding). ${ }^{16}$ Compensated (odds ratio [OR], 2.2) and decompensated (OR, 21.1) cirrhosis were highly significantly predictive of in-hospital mortality in multivariable modeling controlling for age, demographics, insurance status, and hospital features including surgical volume.

In addition, there is evidence that lesser degrees of liver disease may also predict short-term mortality. Benotti and colleagues recently reported outcomes from more than 150,000 patients in the Bariatric Outcome Longitudinal Database. Evidence of liver disease was significantly associated with 30-day mortality after gastric bypass. ${ }^{17}$ In multivariable modeling controlling for body mass index (BMI), age, gender, and cardiopulmonary disease, patients with liver disease were more than twice as likely to die in 30 days compared with those without liver disease (OR, 2.26; 95\% confidence interval, 1.08-4.87). Although these authors used a fairly liberal definition of liver disease including assessment of liver enzymes, imaging, and histologic characteristics to capture patients with a spectrum of NAFLD, liver disease was likely underdiagnosed given the reported prevalence of only $2.4 \%$. The impact of liver disease on long-term surgical outcomes and a more precise understanding of how to risk stratify BS patients with advanced liver disease are important questions that require additional investigation.

\section{Liver Disease in BS Patients Has Been Incompletely Described and Noninvasive Testing Remains Inadequate}

While it is clear that NALFD is common in BS patients, the distribution of each stage (i.e., steatosis, NASH and/or advanced fibrosis, and cirrhosis) and whether there is a consistent pattern of transition between stages to inform prognosis remains uncertain. There are few large studies of BS patients with unbiased biopsy data on which to even make firm statements of prevalence. ${ }^{18,19}$ The most comprehensive prevalence assessment comes from a recent meta-analysis of 12 studies with 1,620 patients who were consecutively biopsied at the time of BS throughout the world. ${ }^{18}$ The prevalence of NASH ranged from $24 \%$ to $98 \%$, and cirrhosis from $0 \%$ to $7 \%$. There are also a limited number of large series of consecutive patients undergoing BS who had intraoperative liver biopsies. In one recent series of 679 patients, $34 \%$ of the patients had $\mathrm{NASH}$, and $7 \%$ had cirrhosis. ${ }^{20}$ Interestingly, stage $\geq 2$ fibrosis was found in $3 \%$ of patients without NAFLD, $18 \%$ of patients with simple steatosis, and $50 \%$ of patients with NASH, indicating that significant fibrotic liver disease may exist in the absence of ongoing inflammation. While risk factors for significant liver pathology in the setting of BS such as diabetes, hyperlipidemia, and geographic location (hinting at other genetic and environmental influences) have been identified, ${ }^{18,21,22}$ none of these can be used alone to accurately predict liver pathology in an individual patient.

Given the important limitations of liver biopsy, including sampling error and the procedure-related morbidity and mortality, there is great interest in replacing biopsy with noninvasive radiographic or serologic testing. To replace the information obtained by histology, noninvasive testing must quantify hepatic fat, differentiate NASH from simple steatosis, and accurately determine fibrosis stage. Unfortunately, there is currently no sufficiently accurate noninvasive tool that can provide this breadth of information. Basic serologic markers of liver injury such as ALT may statistically correlate with hepatic inflammation, but ALT is not sensitive or specific enough to reliably exclude important liver disease. There is clearly a significant burden of NAFLD, NASH, and fibrosis in patients with normal ALTs in the general population ${ }^{23-25}$ and in BS patients. ${ }^{18}$ Even when conservative ALT cutoffs are used $(<19$ $\mathrm{IU} / \mathrm{L}$ for women and $<30 \mathrm{IU} / \mathrm{L}$ for men), significant pathology is not excluded. ${ }^{6,25}$ Thus, a normal ALT cannot be used to justify refraining from performing a liver biopsy at the time of BS. 
Several serologic panels have been developed to quantify hepatic fat (e.g., SteatoTest [BioPredictive, Paris, France], ${ }^{26}$ the fatty liver index, ${ }^{27}$ the lipid accumulation product, ${ }^{28}$ and the NAFLD liver fat score ${ }^{29}$ ), and perhaps, more importantly, differentiate NASH from simple steatosis (e.g., NashTest [BioPredictive], ${ }^{30,31}$ cytokeratin-18, ${ }^{32,33}$ and other clinical and laboratory algorithms $\left.{ }^{34,35}\right) .{ }^{36}$ However, the accuracy of these assays vary significantly between NAFLD cohorts, including BS patients, ${ }^{31,36,37}$ and combinations of biomarkers may be required. ${ }^{38}$ Serologic determinates of fibrosis are perhaps of the greatest clinical significance, and several assays have been designed for and/or tested in NAFLD patients specifically. Panels such as the BARD score, ${ }^{39}$ the FIB-4 index, ${ }^{40}$ and the NAFLD fibrosis score ${ }^{41}$ have been compared in several series, but remain limited by only modest positive and negative predictive values. ${ }^{42,43}$ Among the most commonly referenced is the NAFLD fibrosis score; however, when it was specifically applied to a BS population, only approximately $40 \%$ of patients in the tested cohort could have reasonably avoided a liver biopsy and still had a fairly accurate assessment of fibrosis based on the test result. ${ }^{44}$ Thus, despite the large number of assays available, there is currently no serologic test that can replace the information obtained with liver biopsy.

Radiographic methods are also now commonly used to assess steatosis and fibrosis. Ultrasound is a widely available technique to detect hepatic fat, though traditional ultrasound is unable to quantify fatty infiltration and its sensitivity is lower in patients with $<30 \%$ fat and/or with morbid obesity. ${ }^{45}$ Additional ultrasonographic tools such as the controlled attenuation parameter ${ }^{46}$ and various magnetic resonance (MR) techniques such as chemical shift MR and MR spectroscopy $^{47,48}$ may be among the most sensitive techniques to identify and quantify hepatic fat, ${ }^{47,48}$ but are expensive and/or rarely used clinically. Liver stiffness, as a measure of hepatic fibrosis, can now be assessed with ultrasound or MR elastography, which may be more predictive of advanced fibrosis than serologic markers in this setting. ${ }^{49,50}$ Ultrasound elastography, which was recently approved only in the United States, is the most widely available of these techniques, but has not been extensively tested in the BS population and may not perform as well in the NAFLD population as it does for patients with chronic hepatitis $C$ due to the degree of subcutaneous fat in obese subjects. ${ }^{51,52}$ While imaging techniques will continue to improve and may eventually be the superior approach to quantifying total hepatic fat and/or fibrosis given the sampling error that may occur with biopsy, the major limitation of all available radiographic approaches to date is their inability to detect inflammation, a crucial precursor to the development of progressive disease, including fibrosis.

\section{Impact of Bariatric Surgery on the Natural History of NALFD Is Not Known}

BS has been shown in case-control studies to be associated with a wide variety of significant health benefits including a decrease in all-cause mortality, mortality related to cardiovascular disease, diabetes and cancer, improvement in type 2 diabetes, and improved health-related quality of life. ${ }^{53-59}$ However, despite the fact that the overwhelming majority of BS patients have NAFLD as an obesity-related complication, the impact of BS on the natural history of NAFLD in these patients is unclear. There are no randomized controlled trials that evaluate NAFLD progression, and in the observational data available, follow-up liver biopsies are generally done at variable intervals and only in selected patients. In a recent Cochrane Database Systematic Review, the authors could only conclude that the lack of randomized clinical trial data precluded assessment of the impact of BS on NASH. ${ }^{60}$

There were 21 additional cohort studies included in this Cochrane review, ${ }^{60}$ and studies of BS cohorts with paired liver biopsies have also been compiled in several recent reviews. ${ }^{61-64}$ The vast majority of these studies report reductions in steatosis and lobular inflammation in follow-up compared with intraoperative biopsies, although conclusions about the impact on fibrosis progression are less clear, with several large reports documenting progressive disease. ${ }^{19,65}$ In one of the large prospective cohorts with protocolized liver biopsies 1 and 5 years after BS, the proportion of patients with steatosis and NASH decreased during follow-up; however, fibrosis progression occurred in some patients and the overall mean fibrosis stage increased. ${ }^{65}$ With the variable and generally short-term follow-up of these cohorts, and without a control group of patients who did not undergo BS for comparison, it is difficult to infer the impact of BS on the natural history of NAFLD. There are no large studies with follow-up biopsies over a 5-10 year span after BS that can define the rate of progression between stages in the patients who do or do not lose weight after surgery.

Furthermore, it remains uncertain whether the particular bariatric operation chosen has a significant impact on hepatic effects. It is important to remember that early BS approaches such as jejunoileal bypass frequently resulted in worsening of liver pathology, including reports of precipitating acute liver failure resulting in liver transplant or death. ${ }^{66-73}$ While this procedure is no longer performed, liver failure requiring transplant has also been reported following biliopancreatic diversion, and patients with early rapid weight loss, low albumin levels, and excessive malabsorption and malnutrition may be at particular risk of hepatotoxicity in this setting. ${ }^{73-77}$ In fact, weight loss of greater than $1.6 \mathrm{~kg} /$ week has been associated with increased risk of portal fibrosis. ${ }^{78}$ Thus, the rate of weight loss from any BS approach should be carefully monitored, especially in patients with advanced liver disease. Finally, it is also unknown how manipulation of the hormonal control of weight through various operative approaches and resultant anatomical rearrangements may impact the pathophysiology of NAFLD, though there is emerging evidence that gut peptides such as leptin, ghrelin, glucagon-like peptide-1, and peptide YY play an important role in NAFLD. ${ }^{62,63}$ Additional work is needed to determine whether targeted alteration of these peptide levels may lead to optimization of the surgical approach in patients with NAFLD.

Thus, while BS may be an important tool in the treatment of NASH and NAFLD-related fibrosis, as it is for other obesityrelated comorbidities, no studies are yet available to confirm 
this hypothesis. Despite growing clinical enthusiasm for BS for this indication and the cautious recommendations by some $^{3}$ to proceed with BS when other measures have failed, given the lack of high-quality studies on the topic, the recently released multisociety practice guidelines on the management of NAFLD ${ }^{14}$ appropriately included the following recommendations: “(1) Foregut BS is not contraindicated in otherwise eligible obese individuals with NAFLD and NASH (but without established cirrhosis); (2) The type, safety and efficacy of foregut BS in otherwise eligible individuals with established cirrhosis caused by NAFLD are not established; (3) It is premature to consider foregut BS as an established option specifically to treat NASH." The lack of clinical trial data on the impact of BS on liver-related mortality may be in part due to the large numbers of patients and the relatively long followup needed to observe progression of liver disease and liverrelated patient outcomes. Important causes of death among NAFLD patients including cardiovascular disease and cancer must also be considered in this context, and registry data may be a more realistic tool in the evaluation of the impact of BS on liver-related mortality. However, clinical trials with predetermined protocol liver biopsies are clearly needed.

Finally, understanding the impact of BS specifically on patients with NAFLD-related cirrhosis and indications for liver transplant is also increasingly important. Because of the increased operative risks associated with obesity, many transplant centers have BMI cutoffs above which they will not consider liver transplantation. BS has thus been performed in select patients not only to achieve weight loss but also to render individuals acceptable candidates for life saving liver transplant. ${ }^{79,80}$ In the few series reported, there have been small numbers of liver-related deaths and at least one case in which the Roux-en-Y gastric bypass was reversed due to severe malnutrition. ${ }^{79}$ It is unknown whether BS-associated weight loss may provide an additional benefit of disease stabilization or recompensation in select patients.

\section{Future Directions: An Important Opportunity to Study NAFLD Pathogenesis}

While thousands of patients have undergone BS worldwide, the overwhelming majority of who have NAFLD, there remain significant holes in our understanding of NAFLD in BS patients, including robust prevalence data and the impact of BS on the natural history of the disease. Although cardiovascular disease and diabetes-related complications of obesity have been well studied in BS patients, liver disease remains an under-recognized epidemic in this population.

While liver biopsy has definite limitations in terms of sampling error and the potential for complications, until noninvasive tests are available that can accurately stage fibrosis, differentiate NASH from simple steatosis, and quantify hepatic fatty infiltration, liver biopsy will remain central to the management of these patients. Despite the lack of highly accurate noninvasive markers, the current clinical guidelines do not recommend liver biopsy except in select patients, including those "with NAFLD who are at increased risk to have steatohepatitis and advanced fibrosis." ${ }^{14}$ In addition, their recommendations on the safety and efficacy of BS in this population are contingent upon whether the patient has cirrhosis, which requires accurate assessment of fibrosis stage. BS patients are among those at the highest risk of NAFLD and its complications, and perioperative risk stratification through accurate staging of disease is crucial in avoiding potentially fatal consequences including liver failure. Thus, liver biopsy in this setting, either before BS in patients with clear evidence of advanced liver disease and/or any signs of portal hypertension or at the time of BS in the remaining patients, is an important part of patient management. One unresolved issue in this regard is the relative safety of liver biopsy as a preoperative versus a laparoscopic intraoperative procedure. While NAFLD-specific treatment options remain limited at this time, ${ }^{14}$ important management issues in those with advanced liver disease, including modifying risk factors for disease progression and cardiovascular complications, and screening for HCC and portal hypertension, are crucial goals. As treatment options for patients with NAFLD inevitably improve, just as they finally have for HCV, more aggressive measures to diagnose and stage NAFLD will clearly be indicated and will likely be implemented.

In addition to these benefits to individual patients, accurate characterization of liver histology during BS and subsequently over time may represent an important opportunity to study the pathophysiology of this very complex disease. Coupled with genome-wide association data, such longterm studies may well provide very valuable information about the role of genetics in defining the evolution of NAFLD toward cirrhosis. ${ }^{81}$ Careful examination of the differential impact of specific BS procedures on gut peptides and the evolution of liver disease may offer additional important insights. As the prevalence of obesity and thus the need for BS continues to rise, and NAFLD emerges as a leading indication for liver transplant, liver assessment through biopsy (currently the standard) or eventually through noninvasive markers in BS patients will provide invaluable information for both patients and the field of hepatology. Thus, the recommendation by the LABS Consortium that "consideration should be given to routine liver biopsy during BS and medical follow-up of significant hepatic pathology" is a significant positive step.

\section{References}

1 Charlton MR, Burns JM, Pedersen RA, Watt KD, Heimbach JK, Dierkhising RA. Frequency and outcomes of liver transplantation for nonalcoholic steatohepatitis in the United States. Gastroenterology 2011;141(4):1249-1253

2 Merion RM. Current status and future of liver transplantation. Semin Liver Dis 2010;30(4):411-421

3 Nascimbeni F, Pais R, Bellentani S, et al. From NAFLD in clinical practice to answers from guidelines. J Hepatol 2013;59(4): 859-871

4 Yilmaz Y. Review article: is non-alcoholic fatty liver disease a spectrum, or are steatosis and non-alcoholic steatohepatitis distinct conditions? Aliment Pharmacol Ther 2012;36(9):815-823

5 Singal AK, Guturu P, Hmoud B, Kuo YF, Salameh H, Wiesner RH. Evolving frequency and outcomes of liver transplantation based on etiology of liver disease. Transplantation 2013;95(5):755-760 
6 Kleiner DE, Berk PD, Hsu JY, et al. Hepatic pathology among patients without known liver disease undergoing bariatric surgery: Observations and a perspective from the Longitudinal Assessment of Bariatric Surgery (LABS) Study. Sem Liv Dis 2014; 34(1):98-107

7 Prati D, Taioli E, Zanella A, et al. Updated definitions of healthy ranges for serum alanine aminotransferase levels. Ann Intern Med 2002;137(1):1-10

8 Angulo P. Long-term mortality in nonalcoholic fatty liver disease: is liver histology of any prognostic significance? Hepatology 2010; 51(2):373-375

9 Pagadala MR, McCullough AJ. The relevance of liver histology to predicting clinically meaningful outcomes in nonalcoholic steatohepatitis. Clin Liver Dis 2012;16(3):487-504

10 Younossi ZM, Stepanova M, Rafiq N, et al. Pathologic criteria for nonalcoholic steatohepatitis: interprotocol agreement and ability to predict liver-related mortality. Hepatology 2011;53(6):1874-1882

11 Ekstedt M, Franzén LE, Mathiesen UL, et al. Long-term follow-up of patients with NAFLD and elevated liver enzymes. Hepatology 2006;44(4):865-873

12 Rafiq N, Bai C, Fang Y, et al. Long-term follow-up of patients with nonalcoholic fatty liver. Clin Gastroenterol Hepatol 2009;7(2): 234-238

13 Stepanova M, Rafiq N, Makhlouf $\mathrm{H}$, et al. Predictors of all-cause mortality and liver-related mortality in patients with non-alcoholic fatty liver disease (NAFLD). Dig Dis Sci 2013;58(10):3017-3023

14 Chalasani N, Younossi Z, Lavine JE, et al; American Gastroenterological Association; American Association for the Study of Liver Diseases; American College of Gastroenterology. The diagnosis and management of non-alcoholic fatty liver disease: practice guideline by the American Gastroenterological Association, American Association for the Study of Liver Diseases, and American College of Gastroenterology. Gastroenterology 2012;142(7): 1592-1609

15 Brolin RE, Bradley LJ, Taliwal RV. Unsuspected cirrhosis discovered during elective obesity operations. Arch Surg 1998;133(1):84-88

16 Mosko JD, Nguyen GC. Increased perioperative mortality following bariatric surgery among patients with cirrhosis. Clin Gastroenterol Hepatol 2011;9(10):897-901

17 Benotti P, Wood GC, Winegar DA, et al. Risk factors associated with mortality after Roux-en-Y gastric bypass surgery. Ann Surg 2014; 259(1):123-130

18 Machado M, Marques-Vidal P, Cortez-Pinto H. Hepatic histology in obese patients undergoing bariatric surgery. J Hepatol 2006;45(4): 600-606

19 Kral JG, Thung SN, Biron S, et al. Effects of surgical treatment of the metabolic syndrome on liver fibrosis and cirrhosis. Surgery 2004; 135(1):48-58

20 Bedossa P, Poitou C, Veyrie N, et al. Histopathological algorithm and scoring system for evaluation of liver lesions in morbidly obese patients. Hepatology 2012;56(5):1751-1759

21 Ong JP, Elariny H, Collantes R, et al. Predictors of nonalcoholic steatohepatitis and advanced fibrosis in morbidly obese patients. Obes Surg 2005;15(3):310-315

22 Dixon JB, Bhathal PS, O'Brien PE. Nonalcoholic fatty liver disease: predictors of nonalcoholic steatohepatitis and liver fibrosis in the severely obese. Gastroenterology 2001;121(1):91-100

23 Mofrad P, Contos MJ, Haque M, et al. Clinical and histologic spectrum of nonalcoholic fatty liver disease associated with normal ALT values. Hepatology 2003;37(6):1286-1292

24 Wong VW, Wong GL, Tsang SW, et al. Metabolic and histological features of non-alcoholic fatty liver disease patients with different serum alanine aminotransferase levels. Aliment Pharmacol Ther 2009;29(4):387-396

25 Kunde SS, Lazenby AJ, Clements RH, Abrams GA. Spectrum of NAFLD and diagnostic implications of the proposed new normal range for serum ALT in obese women. Hepatology 2005;42(3): $650-656$
26 Poynard T, Ratziu V, Naveau S, et al. The diagnostic value of biomarkers (SteatoTest) for the prediction of liver steatosis. Comp Hepatol 2005;4:10

27 Bedogni G, Bellentani S, Miglioli L, et al. The Fatty Liver Index: a simple and accurate predictor of hepatic steatosis in the general population. BMC Gastroenterol 2006;6:33

28 Bedogni G, Kahn HS, Bellentani S, Tiribelli C. A simple index of lipid overaccumulation is a good marker of liver steatosis. BMC Gastroenterol 2010;10:98

29 Kotronen A, Peltonen M, Hakkarainen A, et al. Prediction of nonalcoholic fatty liver disease and liver fat using metabolic and genetic factors. Gastroenterology 2009;137(3):865-872

30 Poynard T, Ratziu V, Charlotte F, et al; LIDO Study Group; CYTOL study group. Diagnostic value of biochemical markers (NashTest) for the prediction of non alcoholo steato hepatitis in patients with non-alcoholic fatty liver disease. BMC Gastroenterol 2006;6:34

31 Poynard T, Lassailly G, Diaz E, et al; FLIP consortium. Performance of biomarkers FibroTest, ActiTest, SteatoTest, and NashTest in patients with severe obesity: meta analysis of individual patient data. PLoS ONE 2012;7(3):e30325

32 Cusi K, Chang Z, Harrison S, et al. Limited value of plasma cytokeratin-18 as a biomarker for NASH and fibrosis in patients with non-alcoholic fatty liver disease. J Hepatol 2014;60(1): 167-174

33 Feldstein AE, Wieckowska A, Lopez AR, Liu YC, Zein NN, McCullough AJ. Cytokeratin-18 fragment levels as noninvasive biomarkers for nonalcoholic steatohepatitis: a multicenter validation study. Hepatology 2009;50(4):1072-1078

34 Palekar NA, Naus R, Larson SP, Ward J, Harrison SA. Clinical model for distinguishing nonalcoholic steatohepatitis from simple steatosis in patients with nonalcoholic fatty liver disease. Liver Int 2006;26(2):151-156

35 Shimada M, Kawahara H, Ozaki K, et al. Usefulness of a combined evaluation of the serum adiponectin level, HOMA-IR, and serum type IV collagen 7S level to predict the early stage of nonalcoholic steatohepatitis. Am J Gastroenterol 2007;102(9):1931-1938

36 Castera L, Vilgrain V, Angulo P. Noninvasive evaluation of NAFLD. Nat Rev Gastroenterol Hepatol 2013;10(11):666-675

37 Lassailly G, Caiazzo R, Hollebecque A, et al. Validation of noninvasive biomarkers (FibroTest, SteatoTest, and NashTest) for prediction of liver injury in patients with morbid obesity. Eur J Gastroenterol Hepatol 2011;23(6):499-506

38 Shen J, Chan HL, Wong GL, et al. Non-invasive diagnosis of nonalcoholic steatohepatitis by combined serum biomarkers. J Hepatol 2012;56(6):1363-1370

39 Harrison SA, Oliver D, Arnold HL, Gogia S, Neuschwander-Tetri BA. Development and validation of a simple NAFLD clinical scoring system for identifying patients without advanced disease. Gut 2008;57(10):1441-1447

40 Sterling RK, Lissen E, Clumeck N, et al; APRICOT Clinical Investigators. Development of a simple noninvasive index to predict significant fibrosis in patients with HIV/HCV coinfection. Hepatology 2006;43(6):1317-1325

41 Angulo P, Hui JM, Marchesini G, et al. The NAFLD fibrosis score: a noninvasive system that identifies liver fibrosis in patients with NAFLD. Hepatology 2007;45(4):846-854

42 McPherson S, Stewart SF, Henderson E, Burt AD, Day CP. Simple non-invasive fibrosis scoring systems can reliably exclude advanced fibrosis in patients with non-alcoholic fatty liver disease. Gut 2010;59(9):1265-1269

43 McPherson S, Anstee QM, Henderson E, Day CP, Burt AD. Are simple noninvasive scoring systems for fibrosis reliable in patients with NAFLD and normal ALT levels? Eur J Gastroenterol Hepatol 2013;25(6):652-658

44 Simo KA, McKillop IH, McMillan MT, et al. Does a calculated "NAFLD fibrosis score" reliably negate the need for liver biopsy in patients undergoing bariatric surgery? Obes Surg 2014;24(1): $15-21$ 
45 Schwenzer NF, Springer F, Schraml C, Stefan N, Machann J, Schick F. Non-invasive assessment and quantification of liver steatosis by ultrasound, computed tomography and magnetic resonance. J Hepatol 2009;51(3):433-445

46 Sasso M, Beaugrand M, de Ledinghen V, et al. Controlled attenuation parameter (CAP): a novel VCTE ${ }^{\mathrm{TM}}$ guided ultrasonic attenuation measurement for the evaluation of hepatic steatosis: preliminary study and validation in a cohort of patients with chronic liver disease from various causes. Ultrasound Med Biol 2010;36(11):1825-1835

47 Lee SS, Park SH, Kim HJ, et al. Non-invasive assessment of hepatic steatosis: prospective comparison of the accuracy of imaging examinations. J Hepatol 2010;52(4):579-585

48 van Werven JR, Marsman HA, Nederveen AJ, et al. Assessment of hepatic steatosis in patients undergoing liver resection: comparison of US, CT, T1-weighted dual-echo MR imaging, and pointresolved 1H MR spectroscopy. Radiology 2010;256(1):159-168

49 Kumar R, Rastogi A, Sharma MK, et al. Liver stiffness measurements in patients with different stages of nonalcoholic fatty liver disease: diagnostic performance and clinicopathological correlation. Dig Dis Sci 2013;58(1):265-274

50 Kim D, Kim WR, Talwalkar JA, Kim HJ, Ehman RL. Advanced fibrosis in nonalcoholic fatty liver disease: noninvasive assessment with MR elastography. Radiology 2013;268(2):411-419

51 Petta S, Di Marco V, Cammà C, Butera G, Cabibi D, Craxì A. Reliability of liver stiffness measurement in non-alcoholic fatty liver disease: the effects of body mass index. Aliment Pharmacol Ther 2011;33(12):1350-1360

52 Gaia S, Carenzi S, Barilli AL, et al. Reliability of transient elastography for the detection of fibrosis in non-alcoholic fatty liver disease and chronic viral hepatitis. J Hepatol 2011;54(1):64-71

53 Pontiroli AE, Morabito A. Long-term prevention of mortality in morbid obesity through bariatric surgery. a systematic review and meta-analysis of trials performed with gastric banding and gastric bypass. Ann Surg 2011;253(3):484-487

54 Sjöström L, Narbro K, Sjöström CD, et al; Swedish Obese Subjects Study. Effects of bariatric surgery on mortality in Swedish obese subjects. N Engl J Med 2007;357(8):741-752

55 Adams TD, Gress RE, Smith SC, et al. Long-term mortality after gastric bypass surgery. N Engl J Med 2007;357(8):753-761

56 Schauer PR, Kashyap SR, Wolski K, et al. Bariatric surgery versus intensive medical therapy in obese patients with diabetes. $\mathrm{N} \mathrm{Engl} \mathrm{J}$ Med 2012;366(17):1567-1576

57 Ikramuddin S, Korner J, Lee WJ, et al. Roux-en-Y gastric bypass vs intensive medical management for the control of type 2 diabetes, hypertension, and hyperlipidemia: the Diabetes Surgery Study randomized clinical trial. JAMA 2013;309(21):2240-2249

58 O'Brien PE, Dixon JB, Laurie C, et al. Treatment of mild to moderate obesity with laparoscopic adjustable gastric banding or an intensive medical program: a randomized trial. Ann Intern Med 2006; 144(9):625-633

59 Chang SH, Stoll CR, Song J, Varela JE, Eagon CJ, Colditz GA. The effectiveness and risks of bariatric surgery: an updated systematic review and meta-analysis, 2003-2012. JAMA Surg 2013(Dec)18

60 Chavez-Tapia NC, Tellez-Avila FI, Barrientos-Gutierrez T, MendezSanchez N, Lizardi-Cervera J, Uribe M. Bariatric surgery for nonalcoholic steatohepatitis in obese patients. Cochrane Database Syst Rev 2010;(1):CD007340

61 Dixon JB. Surgical management of obesity in patients with morbid obesity and nonalcoholic fatty liver disease. Clin Liver Dis 2014; 18(1):129-146
62 Verna EC, Berk PD. Role of fatty acids in the pathogenesis of obesity and fatty liver: impact of bariatric surgery. Semin Liver Dis 2008; 28(4):407-426

63 Rabl C, Campos GM. The impact of bariatric surgery on nonalcoholic steatohepatitis. Semin Liver Dis 2012;32(1):80-91

64 Hafeez S, Ahmed MH. Bariatric surgery as potential treatment for nonalcoholic fatty liver disease: a future treatment by choice or by chance? J Obes 2013;2013:839275

65 Mathurin P, Hollebecque A, Arnalsteen L, et al. Prospective study of the long-term effects of bariatric surgery on liver injury in patients without advanced disease. Gastroenterology 2009;137(2):532-540

66 Drenick EJ, Simmons F, Murphy JF. Effect on hepatic morphology of treatment of obesity by fasting, reducing diets and small-bowel bypass. N Engl J Med 1970;282(15):829-834

67 Marubbio AT Jr, Buchwald H, Schwartz MZ, Varco R. Hepatic lesions of central pericellular fibrosis in morbid obesity, and after jejunoileal bypass. Am J Clin Pathol 1976;66(4):684-691

68 Campbell JM, Hunt TK, Karam JH, Forsham PH. Jejunoileal bypass as a treatment of morbid obesity. Arch Intern Med 1977;137(5): 602-610

69 Vyberg M, Ravn V, Andersen B. Pattern of progression in liver injury following jejunoileal bypass for morbid obesity. Liver 1987; 7(5):271-276

70 Hocking MP, Duerson MC, O'Leary JP, Woodward ER. Jejunoileal bypass for morbid obesity. Late follow-up in 100 cases. N Engl J Med 1983;308(17):995-999

71 Marubbio AT, Rucker RD Jr, Schneider PD, Horstmann JP, Varco RL, Buchwald $\mathrm{H}$. The liver in morbid obesity and following bypass surgery for obesity. Surg Clin North Am 1979;59(6):1079-1093

72 DeWind LT, Payne JH. Intestinal bypass surgery for morbid obesity. Long-term results. JAMA 1976;236(20):2298-2301

73 Geerts A, Darius T, Chapelle T, et al. The multicenter Belgian survey on liver transplantation for hepatocellular failure after bariatric surgery. Transplant Proc 2010;42(10):4395-4398

74 Antal SC. Prevention and reversal of liver damage following biliopancreatic diversion for obesity. Obes Surg 1994;4(3): 285-290

75 D'Albuquerque LA, Gonzalez AM, Wahle RC, de Oliveira Souza E, Mancero JM, de Oliveira e Silva A. Liver transplantation for subacute hepatocellular failure due to massive steatohepatitis after bariatric surgery. Liver Transpl 2008;14(6):881-885

76 Castillo J, Fábrega E, Escalante CF, et al. Liver transplantation in a case of steatohepatitis and subacute hepatic failure after biliopancreatic diversion for morbid obesity. Obes Surg 2001;11(5): 640-642

77 Grimm IS, Schindler W, Haluszka O. Steatohepatitis and fatal hepatic failure after biliopancreatic diversion. Am J Gastroenterol 1992;87(6):775-779

78 Andersen T, Gluud C, Franzmann MB, Christoffersen P. Hepatic effects of dietary weight loss in morbidly obese subjects. J Hepatol 1991;12(2):224-229

79 Al-Nowaylati AR, Al-Haddad BJ, Dorman RB, et al. Gastric bypass after liver transplantation. Liver Transpl 2013;19(12):1324-1329

80 Lin MY, Tavakol MM, Sarin A, et al. Laparoscopic sleeve gastrectomy is safe and efficacious for pretransplant candidates. Surg Obes Relat Dis 2013;9(5):653-658

81 Speliotes EK, Yerges-Armstrong LM, Wu J, et al; NASH CRN; GIANT Consortium; MAGIC Investigators; GOLD Consortium. Genomewide association analysis identifies variants associated with nonalcoholic fatty liver disease that have distinct effects on metabolic traits. PLoS Genet 2011;7(3):e1001324 